https://helda.helsinki.fi

\title{
Radiatively induced Fermi scale and unification
}

\section{Alanne, Tommi}

2016-05-09

Alanne , T , Meroni , A, Sannino , F \& Tuominen, K 2016 , ' Radiatively induced Fermi scale and unification ' , Physical Review D , vol. 93 , no. 9 , 091701 . https://doi.org/10.1103/PhysRevD.93.091701

http://hdl.handle.net/10138/185065

https://doi.org/10.1103/PhysRevD.93.091701

cc_by

publishedVersion

Downloaded from Helda, University of Helsinki institutional repository.

This is an electronic reprint of the original article.

This reprint may differ from the original in pagination and typographic detail.

Please cite the original version. 
PHYSICAL REVIEW D 93, 091701(R) (2016)

\title{
Radiatively induced Fermi scale and unification
}

\author{
Tommi Alanne, ${ }^{*}$ Aurora Meroni, ${ }^{\dagger}$ and Francesco Sannino \\ $C P^{3}$-Origins \& the Danish Institute for Advanced Study Danish IAS, University of Southern Denmark, \\ Campusvej 55, DK-5230 Odense M, Denmark
}

\author{
Kimmo Tuominen ${ }^{\S}$ \\ Department of Physics, University of Helsinki, \& Helsinki Institute of Physics, P.O. Box 64,
} FI-00014 University of Helsinki, Finland

(Received 11 November 2015; published 9 May 2016)

\begin{abstract}
We consider extensions of the Standard Model in which the hierarchy between the unification and the Fermi scale emerges radiatively. Within the Pati-Salam framework, we show that it is possible to construct a viable model where the Higgs is an elementary pseudo-Goldstone boson, and the correct hierarchy is generated.
\end{abstract}

DOI: 10.1103/PhysRevD.93.091701

\section{INTRODUCTION}

The unification paradigm has motivated several extensions of the Standard Model (SM). Two time-honored unification schemes stand out: the Georgi-Glashow [1] and the Pati-Salam [2]. The former minimally unifies color and electroweak (EW) symmetries in a gauge group such as $\mathrm{SU}(5)$ or $\mathrm{SO}(10)[3,4]$ and predicts gauge-mediated proton decay. The current lower bound on the proton lifetime, set by SuperKamiokande to $\tau>10^{34} \mathrm{y}$ [5], translates into a lower bound on the unification scale of the order of $\gtrsim 10^{15} \mathrm{GeV}$, which is also the natural scale for the realization of type-I seesaw models [6-10]. In the PatiSalam model one unifies quarks and leptons by promoting the lepton number to the fourth color. In this scheme the proton does not decay via gauge interactions, and therefore the previous bound does not apply. On the other hand, spin-one leptoquarks would mediate the $K_{\mathrm{L}} \rightarrow \mu^{ \pm} e^{\mp}$ decay that is severely constrained by experiments leading to a lower bound [11] $M>1.5 \times 10^{6} \mathrm{GeV}$ on their masses. Consequently, the lower bound on the Pati-Salam unification is $\Lambda_{\mathrm{PS}}>1.9 \times 10^{6} \mathrm{GeV}$.

From experiments it is, therefore, clear that there are several well-separated energy scales involved in unified theories. At the very least, one needs the Fermi scale (where the EW symmetry breaks) and the scale where the theory unifies. These two scales are typically modelled via ad hoc scalar sectors.

It would seem appealing to us if there were just one common scale for the full scalar sector, with the Fermi scale emerging radiatively. We will show that such a scenario arises when the Higgs is an elementary pseudo-Goldstone boson (pGB) [12]. This opens the way to alternative scalar

\footnotetext{
*alanne@cp3.sdu.dk

†meroni@cp3.sdu.dk

sannino@cp3.dias.sdu.dk

§immo.i.tuominen@helsinki.fi
}

sector constructions in unified schemes that we believe to be more natural than traditional ones.

Alternative unification scenarios featuring pGB Higgses, such as the GIFT mechanism (Goldstones instead of finetuning) [13], have been discussed in the literature. The main difference and strength of our approach, with respect to previous works, are self-consistency and minimality. In fact, the electroweak and top sector of the SM radiatively determine the vacuum alignment of the theory and dynamically generate the Higgs mass.

As a relevant example we will implement our idea within the Pati-Salam unification framework. Similar constructions à la Georgi-Glashow are left to future investigations.

\section{A MINIMAL PATI-SALAM SETUP}

We start with the elementary Goldstone Higgs (EGH) scenario introduced in Refs. $[12,14]$ according to which the Higgs doublet lives in the $\mathrm{SU}(4) / \mathrm{Sp}(4)$ coset, and the EW symmetry, $\mathrm{SU}(2)_{\mathrm{L}} \times \mathrm{U}(1)_{Y}$, is embedded in $\mathrm{SU}(4)$.

In comparison with the fundamental composite (Goldstone) Higgs idea [15-17], the major differences are that the elementary case is amenable to perturbation theory, it is straightforward to endow the SM fermions with mass terms, and it is possible to consider unified extensions.

As a minimal unified extension of the EGH, we consider here the Pati-Salam framework. We show that the Fermi scale is radiatively induced while the unification scale is held fixed to a phenomenologically viable value. Explicitly, we first extend the color group to an $\mathrm{SU}(4)_{\mathrm{PS}}$ of leptocolor. Differently from the original Pati-Salam construction, we extend the global symmetry of the Higgs sector to be $\mathrm{SU}(4)_{\chi}$ rather than $\mathrm{SU}(2)_{\mathrm{L}} \times \mathrm{SU}(2)_{\mathrm{R}}$. We indicate the full non-Abelian structure by $G=\mathrm{SU}(4)_{\chi} \times \mathrm{SU}(4)_{\mathrm{PS}}$. For simplicity, we consider only one generation of fermions and gauge the $\mathrm{EW} \mathrm{SU}(2)_{\mathrm{L}} \times \mathrm{U}(1)_{Y}$ subgroup of $\mathrm{SU}(4)_{\chi}$. In the original work [2], the full $\mathrm{SU}(2)_{\mathrm{L}} \times$ $\mathrm{SU}(2)_{\mathrm{R}}$ was gauged; the consequences of adapting this to the present model will be considered elsewhere. 
ALANNE, MERONI, SANNINO, and TUOMINEN

We want to identify a minimal number of scalar degrees of freedom to achieve the desired symmetry-breaking pattern and the radiative generation of the Fermi scale. We find this set to consist of two scalar multiples with the following assignments with respect to $G=\mathrm{SU}(4)_{\chi} \times$ $\mathrm{SU}(4)_{\mathrm{PS}}{ }^{1}$ :

$$
M \sim(6,1), \quad P \sim(1,15) .
$$

The most general renormalizable scalar potential then reads

$$
V=V_{M}+V_{P}+V_{M P}
$$

where

$$
\begin{aligned}
V_{M} & =\frac{1}{2} m_{M}^{2} \operatorname{Tr}\left[M^{\dagger} M\right]+\frac{\lambda_{M}}{4} \operatorname{Tr}\left[M^{\dagger} M\right]^{2}, \\
V_{P} & =m_{P}^{2} \operatorname{Tr}\left[P^{2}\right]+\lambda_{P 1} \operatorname{Tr}\left[P^{2}\right]^{2}+\lambda_{P 2} \operatorname{Tr}\left[P^{4}\right], \\
V_{M P} & =\frac{\lambda_{M P}}{2} \operatorname{Tr}\left[M^{\dagger} M\right] \operatorname{Tr}\left[P^{2}\right] .
\end{aligned}
$$

We parametrize $P$ as

$$
P=p_{a} T^{a}
$$

and $M$ as

$$
M=\left[\frac{\sigma}{2}+\mathrm{i} \sqrt{2} \Pi_{i} X^{i}\right] E,
$$

where the index $a$ runs over the 15 generators of $\mathrm{SU}(4)_{\mathrm{PS}}$ and $i$ over the five broken generators $X^{i}$ of $S U(4)_{\chi}$, and $E$ is a fully antisymmetric matrix. The vacuum expectation value (VEV) of $M$ is then given by $\langle M\rangle=\frac{v_{0}}{2} E$.

We have one more scalar that is required to acquire a VEV, $\langle P\rangle=b_{0} T^{15}$, to break the leptocolor group to $\mathrm{SU}(3)_{\mathrm{c}} \times \mathrm{U}(1)_{\mathrm{B}-\mathrm{L}}$. Minimizing the tree-level potential we obtain

$$
\begin{aligned}
b_{0}^{2} & =\frac{6 \lambda_{M P} m_{M}^{2}-12 \lambda_{M} m_{P}^{2}}{\lambda_{M}\left(12 \lambda_{P 1}+7 \lambda_{P 2}\right)-3 \lambda_{M P}^{2}}, \\
v_{0}^{2} & =\frac{6 \lambda_{M P} m_{P}^{2}-\left(12 \lambda_{P 1}+7 \lambda_{P 2}\right) m_{M}^{2}}{\lambda_{M}\left(12 \lambda_{P 1}+7 \lambda_{P 2}\right)-3 \lambda_{M P}^{2}} .
\end{aligned}
$$

These coupled expressions for the VEVs of the two scalar fields would require couplings that are vastly different in value to be able to accommodate simultaneously the unification and the Fermi scale. We will show in the next section that the Fermi scale can, de facto, be radiatively generated.

\footnotetext{
${ }^{1} \mathrm{We}$ use the adjoint representation to be able to preserve the $\mathrm{U}(1)_{\mathrm{B}-\mathrm{L}}$ subgroup of $\mathrm{SU}(4)_{\mathrm{PS}}$ in addition to $\mathrm{SU}(3)_{\mathrm{c}}$.
}

PHYSICAL REVIEW D 93, 091701(R) (2016)

\section{RADIATIVE FERMI SCALE}

The symmetry-breaking pattern $\mathrm{SU}(4) \rightarrow \mathrm{Sp}(4)$ has been studied at length in the literature $[17,18]$. The EW gauge group can be embedded in SU(4) in different ways with respect to the vacuum. We parametrize this freedom by an angle $\theta$. The matrix $E$ in Eq. (5) is correspondingly replaced by $E_{\theta}$,

$$
E_{\theta}=\sin \theta\left(\begin{array}{cc}
0 & 1 \\
-1 & 0
\end{array}\right)+\cos \theta\left(\begin{array}{cc}
\mathrm{i} \sigma_{2} & 0 \\
0 & -\mathrm{i} \sigma_{2}
\end{array}\right) \text {. }
$$

For $\theta=0$, the EW symmetry remains unbroken and for $\theta=\pi / 2$ the EW symmetry directly breaks to $\mathrm{U}(1)_{Q}$. The specific value of $\theta$ must be determined dynamically once the EW and top quark quantum corrections are taken into account. As shown in Refs. [12,14], these corrections favor small values of $\theta$, and consequently the Fermi scale, $v_{w}=v_{0} \sin \theta$, lies well below the spontaneous symmetry-breaking scale $v_{0}$. Furthermore, the radiative corrections provide a mass for the pGB Higgs via the Coleman-Weinberg mechanism.

Following Ref. [12], in the $\overline{\mathrm{MS}}$ scheme, the one-loop potential is

$$
\delta V=\frac{1}{64 \pi^{2}} \operatorname{Str}\left[\mathcal{M}^{4}(\Phi)\left(\log \frac{\mathcal{M}^{2}(\Phi)}{\mu_{0}^{2}}-C\right)\right],
$$

where $\mathcal{M}(\Phi)$ is the tree-level mass matrix of the scalar fields that we denote collectively as $\Phi$, computed using the background field method. The supertrace Str, is defined by

$$
\mathrm{Str}=\sum_{\text {scalars }}-2 \sum_{\text {fermions }}+3 \sum_{\text {vectors }}
$$

We have $C=3 / 2$ for scalars and fermions, while $C=5 / 6$ for the gauge bosons and we include contributions from the EW gauge bosons and the top quark.

We fix the renormalization scale by requiring that the VEV $v=\langle\sigma\rangle$ is given by the tree-level value $v_{0}$ while the one for $\left\langle p_{15}\right\rangle=b$ is determined by minimizing the full one-loop potential along with the dynamical value of $\theta$.

Three states have the quantum numbers of the Higgs, i.e. $\Pi_{4}, \sigma$ and $p_{15}$. In order to find the actual alignment of the vacuum, we minimize the full potential at the one-loop level. We emphasize that the specific value of $\theta$ at the minimum is dynamically generated via quantum effects. In particular, the small value of $\theta$ stems from the top-Yukawa interaction and the EW gauge sector. Furthermore, the experimental constraints coming from the measurement of the Higgs mass are a decisive ingredient in order to constrain the parameter space. The Higgs phenomenology constraints were investigated in Refs. [12,14], where it was shown that the EGH paradigm successfully reproduces the phenomenological success of the SM, including the precision parameters. The same analysis applies here. 


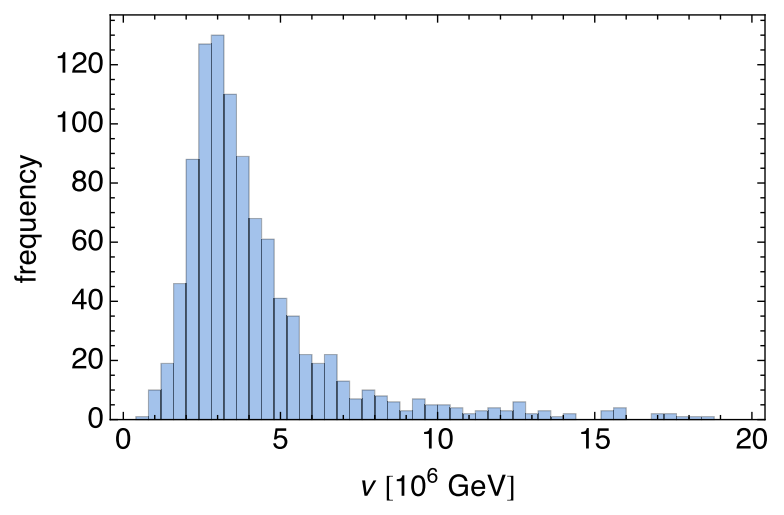

FIG. 1. Distribution of values of $v$ with $b=2.5 \times 10^{6} \mathrm{GeV}$.

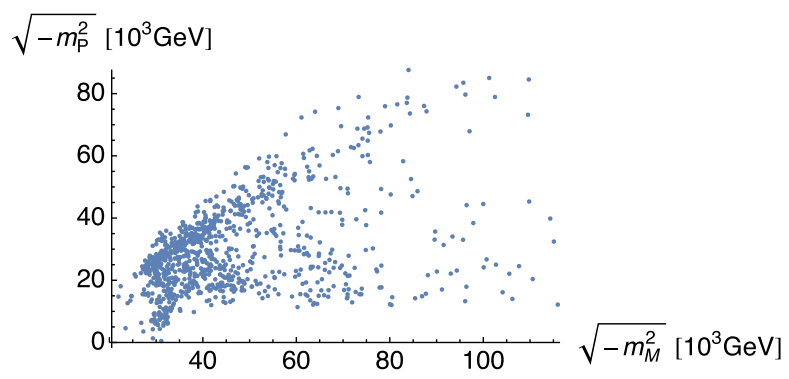

FIG. 2. Distribution of $\sqrt{-m_{M}^{2}}$ vs $\sqrt{-m_{P}^{2}}$.

In the numerical analysis, we show that a small value of $\theta$ is preferred, and therefore the observed Higgs is mostly the pGB $\Pi_{4}$ with a tiny admixture of $\sigma$. We explore the parameter space $\left(b, v, \theta, \lambda_{M}, \lambda_{M P}, \lambda_{P 1}, \lambda_{P 2}\right)$ by assuming the leptocolor breaking scale to be just above the experimental bound, i.e. $b=2.5 \times 10^{6} \mathrm{GeV},{ }^{2}$ and we check that the tree-level potential, Eq. (2), is bounded from below. In Fig. 1 we show the resulting values of the scale $v$ of the global symmetry breaking. The preferred values of $v$ are roughly of the order of $b$, and this feature is reflected also in the Lagrangian mass parameters as shown in Fig. 2. To produce the correct Fermi scale this implies that small values of the angle $\theta$ are favored.

We also find the distribution of quartic couplings shown in Fig. 3. We see that the values of the quartic couplings are overall small, less than $10^{-2}$ for all scanned points; in particular $\lambda_{M P} \sim \mathcal{O}\left(10^{-4}\right)$. Generally we can understand this as follows. The minimization procedure gives a relation between the couplings of the scalar potential and the vacuum angle $\theta$. In the limit of equal self-couplings ${ }^{3}$ and $v=b$, we find $\lambda \sim \sin ^{2} \theta$. Furthermore, we fix the

\footnotetext{
${ }^{2}$ We have also explored regions of parameter space where $b$ is allowed to be significantly larger, e.g. $b \sim \mathcal{O}\left(10^{10} \mathrm{GeV}\right)$. We find that it is still possible to have $b \sim v$ with $v_{\mathrm{w}}$ emerging as a radiatively generated scale.

${ }^{3}$ In the general case the minimization requirement produces a nonlinear logarithmic equation which cannot be solved analytically.
}

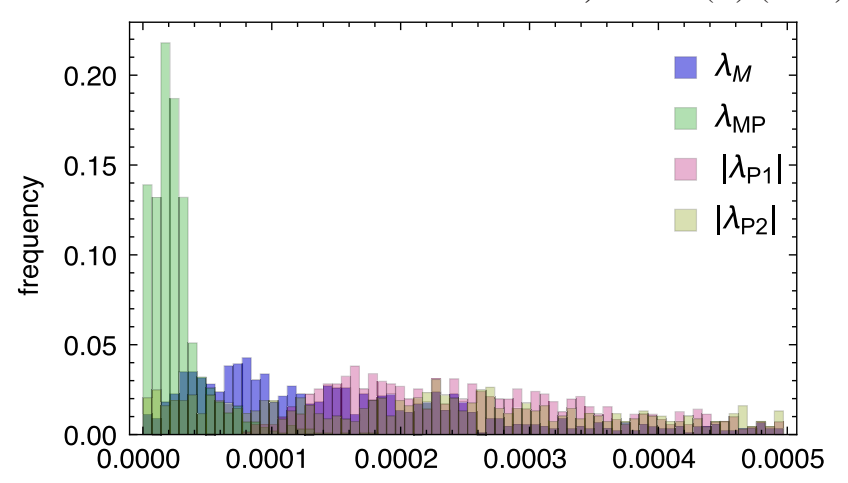

FIG. 3. Distribution of the quartic scalar couplings of 1000 viable scanned points with $b=2.5 \times 10^{6} \mathrm{GeV}$.

mass of the lightest scalar to be $125 \mathrm{GeV}$. This is sensitive in particular to the coupling $\lambda_{M P}$, explaining the restriction on its values as shown in Fig. 3.

To estimate the sensitivity of the electroweak symmetrybreaking scale with respect to the couplings, we determine

$$
\Delta_{\lambda_{i}}=\left|\frac{\lambda_{i}}{v} \frac{\partial v}{\partial \lambda_{i}}\right|
$$

where $i=M, P, P 1, P 2$. The combined estimate of the fine-tuning with respect to the quartic couplings is then given by

$$
\Delta=\sqrt{\sum_{i} \Delta_{\lambda_{i}}^{2}}
$$

We find $\Delta<1$ for the range of parameters we have considered. This implies that the electroweak scale is achieved with a moderate level of fine-tuning.

The numerical analysis demonstrates that it is possible to find a viable parameter space satisfying all phenomenological constraints in this model. ${ }^{4}$

\section{CONCLUSIONS AND OUTLOOK}

We have shown that a dynamical mechanism, in the form of radiative corrections, can generate the desired hierarchy between the EW and unification scales.

The natural starting point is the EGH model with the symmetry-breaking pattern $\mathrm{SU}(4)_{\chi} \rightarrow \mathrm{Sp}(4)_{\chi}$. Of the five GBs, three become the longitudinal components of the EW gauge bosons while the fourth one, via mixing with the radial mode, is identified with the observed Higgs particle. The remaining GB, $\Pi_{5}$, can be a dark matter candidate [12].

\footnotetext{
${ }^{4}$ Because of the relatively large unification scale, smaller values of the scalar couplings are needed, and this reduces the parameter space of the theory. This in not in contradiction with the results of Ref. [14] that favored a lower scale for the VEV of the $\sigma$ field and the scalar couplings were allowed to span a much larger parameter space.
} 
ALANNE, MERONI, SANNINO, and TUOMINEN

The heaviest states in the spectrum are the massive spinone leptoquarks, whose masses are constrained by experiments to be above $10^{3} \mathrm{TeV}$. The heavy scalars have masses $\mathcal{O}(10-100 \mathrm{TeV})$. The lightest states of the spectrum are the pGBs.

In this scenario all scalar self-couplings are generally very small and hence lead to testable consequences. In practice, measuring the trilinear Higgs coupling at the LHC [19] is sufficient to constrain this framework.

A more in-depth analysis of dark matter can be done following Ref. [12]. This would require adding a small $\mathrm{SU}(4)_{\chi}$-breaking mass for $\Pi_{5}$.

Another interesting avenue to explore is neutrino masses and mixings. One can, for example, accommodate right-handed neutrinos into the fundamental representation of $\mathrm{SU}(4)_{\chi}$. This would naturally lead to Dirac masses for neutrinos. More generally, various seesaw scenarios can also be realized in this setup and studied in connection with collider phenomenology [20]. For
PHYSICAL REVIEW D 93, 091701(R) (2016)

example, one could gauge the full chiral symmetry subgroup $\mathrm{SU}(2)_{\mathrm{L}} \times \mathrm{SU}(2)_{\mathrm{R}}$ [or even the entire $\mathrm{SU}(4)_{\chi}$ ]. This would allow one to implement the type-II seesaw mechanism [21-23].

In addition, the scalar sectors could also drive cosmic inflation [24-26] known to also prefer small scalar selfcouplings.

It is therefore clear from the above that new prospects are ready to be explored within the radiatively generated Fermi scale in unification scenarios.

\section{ACKNOWLEDGMENTS}

We thank Borut Bajc for enlightening discussions. The $\mathrm{CP}^{3}$-Origins centre is partially funded by the Danish National Research Foundation, Grant No. DNRF90. T. A. would like to thank the Finnish Cultural Foundation for financial support. K. T. acknowledges support from the Academy of Finland, Project No. 267842.
[1] H. Georgi and S. L. Glashow, Phys. Rev. Lett. 32, 438 (1974).

[2] J. C. Pati and A. Salam, Phys. Rev. D 10, 275 (1974); 11, 703(E) (1975).

[3] H. Georgi, in Particles and Fields, edited by C. E. Carlson (AIP, New York, 1975), p. 575.

[4] H. Fritzsch and P. Minkowski, Ann. Phys. (N.Y.) 93, 193 (1975).

[5] H. Nishino et al. (Super-Kamiokande Collaboration), Phys. Rev. D 85, 112001 (2012).

[6] P. Minkowski, Phys. Lett. 67B, 421 (1977).

[7] T. Yanagida, Conf. Proc. C 7902131, 95 (1979).

[8] P. Ramond, arXiv:hep-ph/9809459.

[9] M. Gell-Mann, P. Ramond, and R. Slansky, Conf. Proc. C 790927, 315 (1979).

[10] R. N. Mohapatra and G. Senjanovic, Phys. Rev. Lett. 44, 912 (1980).

[11] M. K. Parida, R. L. Awasthi, and P. K. Sahu, J. High Energy Phys. 01 (2015) 045.

[12] T. Alanne, H. Gertov, F. Sannino, and K. Tuominen, Phys. Rev. D 91, 095021 (2015).

[13] K. Inoue, A. Kakuto, and H. Takano, Prog. Theor. Phys. 75, 664 (1986).
[14] H. Gertov, A. Meroni, E. Molinaro, and F. Sannino, Phys. Rev. D 92, 095003 (2015).

[15] D. B. Kaplan and H. Georgi, Phys. Lett. 136B, 183 (1984).

[16] D. B. Kaplan, H. Georgi, and S. Dimopoulos, Phys. Lett. 136B, 187 (1984).

[17] G. Cacciapaglia and F. Sannino, J. High Energy Phys. 04 (2014) 111.

[18] T. Appelquist, P. S. Rodrigues da Silva, and F. Sannino, Phys. Rev. D 60, 116007 (1999).

[19] V. Barger, L. L. Everett, C. B. Jackson, and G. Shaughnessy, Phys. Lett. B 728, 433 (2014).

[20] F. F. Deppisch, P. S. Bhupal Dev, and A. Pilaftsis, New J. Phys. 17, 075019 (2015).

[21] M. Magg and C. Wetterich, Phys. Lett. 94B, 61 (1980).

[22] J. Schechter and J. W. F. Valle, Phys. Rev. D 22, 2227 (1980).

[23] R. N. Mohapatra and G. Senjanovic, Phys. Rev. D 23, 165 (1981).

[24] A. A. Starobinsky, Pis'ma Zh. Eksp. Teor. Fiz. 30, 719 (1979) [JETP Lett. 30, 682 (1979)].

[25] A. H. Guth, Phys. Rev. D 23, 347 (1981).

[26] A. D. Linde, Phys. Lett. 108B, 389 (1982). 